\title{
ZOOARQUEOLOGÍA EN LA LLANURA ALUVIAL DEL PARANÁ: EL SITIO CAMPO BINAGHI DESDE UNA PERSPECTIVA REGIONAL
}

\section{ZOOARCHAELOGY IN THE ALUVIAL PLAIN OF THE PARANÁ RI- VER: SITE “CAMPO BINAGHI" FROM A REGIONAL PERSPECTIVE”}

\author{
Julieta Sartori ${ }^{1}$ y Laura Pérez Jimeno ${ }^{2}$ \\ 1 CONICET-INAPL-UNR-FUNDARQ. E-mail: Julisartori@gmail.com \\ 2UNR-INAPL. E-mail: lperezjimeno@yahoo.com.ar
}

Presentado el: 17/04/2012/ - Aceptado el: 16/10/2012

\begin{abstract}
Resumen
En el presente trabajo se dan a conocer los resultados del análisis de los restos arqueofaunísticos provenientes del sitio Campo Binaghi, ubicado en la llanura aluvial del Paraná medio (Las Toscas, Prov. de Santa Fe). Fueron identificadas distintas especies de cérvidos, roedores, dasipódidos, peces y reptiles, de los cuales se discute aquí su ingreso al registro, así como también la importancia relativa de cada taxa. Para establecer estos aspectos se realizó, por un lado, el relevamiento de los agentes tafonómicos que podrían estar incidiendo en la muestra, y por otro, el de los factores relacionados con el tipo de procesamiento (eg. huellas, tipo y estado de fractura). Si bien Campo Binaghi se encuentra dentro de la misma ecoregión que otros sitios estudiados, como Cerro Aguará y Barrancas del Paranacito, se haya emplazado en un área geomorfológicamente más alta y abierta, lo que facilitaría el acceso a otro tipo de recursos propios de estos ambientes. El conocer el tipo de explotación faunistica que habrían realizado los grupos cazadores-recolectores que habitaron Campo Binaghi es de gran relevancia, ya que permitirá compararla con la de aquellos contextos que se encuentran próximos, pudiendo ampliar de este modo el conocimiento acerca de la utilización del espacio y los recursos de la zona en el pasado.
\end{abstract}

Palabras claves: Llanura aluvial del Paraná, Zooarqueología, Cazadores-recolectores

\begin{abstract}
This paper analyzes the archaeofaunal material recovered of Campo Binaghi site, which is located at the Alluvial Plain of Paraná river (Las Toscas, province of Santa Fe). Several species of fishes, armadillos, rodents, deers and reptile were identify upon which discuss its introduction in the archaeological recover and the relative importance of each one. For this aims we realized the observation of taphonomics agents that could be affecting the sample and aspects related with the processing of the prey (eg. cut marks). Campo Binaghi have been found in the same eco-region as other sites, like Cerro Aguará and Barrancas del Paranacito, but the first one is located in higher and wider
\end{abstract}


area than latters two. These characteristics produce the access to more and different resources that are specific of this kind of environments. It is relevant to have known the patron of exploitation of faunistic resources by hunter-gatherer in this zone in order to compare CB with other records. This comparative analysis allow us expand the knowledge about the utilization of space and resources during the past.

Keywords: Alluvial Plain of Paraná river, Zooarchaeology, Hunter-gatherer

\section{Introducción}

El presente trabajo se centra en el análisis de los materiales arqueofaunísticos provenientes del sitio Campo Binaghi (CB), ubicado al noreste de la provincia de Santa Fe (Las Toscas, Dpto. General Obligado). Este sitio posee algunas particularidades respecto de su emplazamiento, ya que se localiza en un sector más abierto y alto que otros sitios de la zona, como Barrancas del Paranacito (BP) (Lafón 1971, 1972; Pérez Jimeno 1996, 2005, 2007) y Cerro Aguará (CA) ( Pérez Jimeno 2007) (Figura 1). Estas localidades arqueológicas son de gran relevancia por su densidad y diversidad de materiales arqueológicos, principalmente, cerámica utilitaria, restos arqueofaunísticos, instrumental óseo y desechos de manufactura (Pérez Jimeno 2007).

Estos últimos dos sitios comenzaron a ser estudiados con el objetivo general de analizar la variabilidad del registro arqueológico para inferir la forma en que las distintas sociedades utilizaron el espacio y los recursos. Para esto se utilizó la heterogénea información que se encontraba disponible para la región hasta entonces (Lafón 1971,1972; Schmitz et al. 1972; Ruggeroni 1975), cruzándose los datos existentes con los generados en la localidad de Florencia.

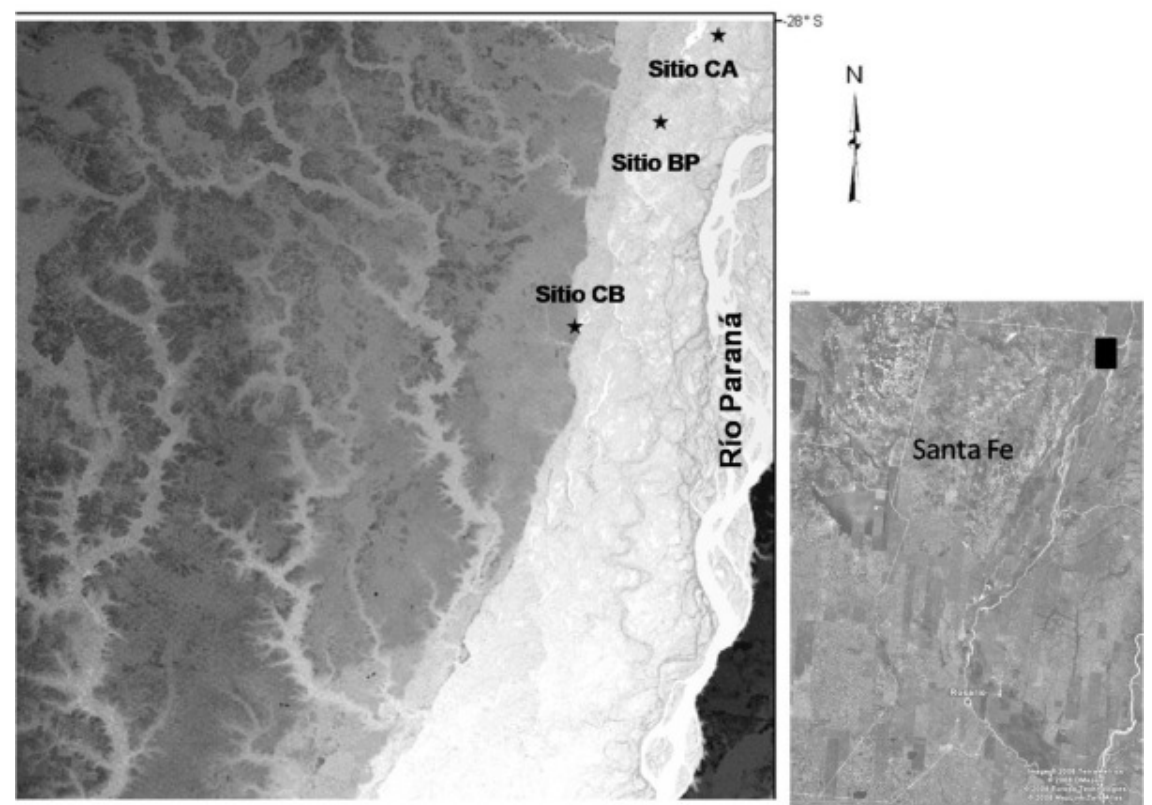

Figura 1. Localización de Campo Binaghi, Barrancas del Paranacito y Cerro Aguará. La escala de grises refleja la altitud topográfica 
De ese modo se propuso un modelo de movilidad y utilización del espacio que en forma sintética plantea que: la llanura aluvial del Paraná Medio durante el Holoceno tardío, particularmente entre 1500 y 500 años AP, habría estado ocupada por grupos humanos semi-sedentarios, vinculados estrechamente a ambientes lénticos generados por la dinámica propia del río Paraná. Estos grupos contaban con tecnología cerámica, ósea y lítica (en algunos sectores), y basaban su subsistencia en la pesca, la recolección -principalmente de moluscos- y la caza.

Según las características ambientales, y acorde con los análisis zooarqueológicos intersitio, se infiere que habrían explotado intensivamente este espacio regional durante los períodos de bajante del río Paraná, a fines del invierno y principios de la primavera. Es posible que en algunas oportunidades se prolongara su permanencia, hasta comienzos del nuevo período de inundación, cuando se fraccionaban y desplazaban hacia las tierras altas de la margen izquierda del río Paraná o hacia el oeste, a áreas ecotonales para aprovechar otros recursos diferentes a los propios del humedal.

Si bien los materiales faunísticos de CB provienen de un sondeo, el análisis preliminar permite comenzar a realizar estudios comparativos con los sitios mencionados y realizar inferencias respecto de la utilización de los recursos en el pasado, aunque las mismas deberán ser contrastadas con una muestra más amplia en el futuro. En este sentido, teniendo en cuenta el lugar de emplazamiento de $\mathrm{CB}$, es posible que su registro arqueológico esté reflejando el momento de fraccionamiento del grupo antes referido, y una estrategia de explotación de recursos y utilización del espacio distinta a las observadas en CA y BP.

Como se observó en este análisis, es esperable que el/los grupo/s humano/s que habitaron $\mathrm{CB}$, hayan explotado taxa propios de áreas topográficamente más altas y abiertas que las de los sitios BP y CA, probablemente durante los momentos de inundación, en el que estos grupos humanos prefirieron aprovechar estos espacios altos (ver Pérez Jimeno 2007).

\section{Marco ambiental y disponibilidad de recursos}

El área en que se localiza el sitio CB pertenece zoogeográficamente a la Región Neotropical, Subregión Guayanobrasileña, subdistrito chaqueño, el cual es muy rico por su alta biodiversidad (Cabrera y Yepes 1940, SPANP 1997). Dentro de la gran cantidad de especies que posee puede mencionarse la presencia de: monos carayá (Aloatta caraya), comadreja overa (Didelphis albiventris) y colorada (Lutreolina crassicaudata), tatú negro o mulita grande (Dasypus hybridus), oso hormiguero (Myrmecophaga tridactyla), aguará guazú (Chrysocyon brachyurus), zorro gris (Lycalopex gymnocercus) y zorro de monte (Cerdocyon thous), lobito de río (Lontra longicaudis), felinos como el gato montés (Oncifelis geoffroyi) y roedores como el carpincho (Hydrochoerus hydrochaeris), el coipo (Myocastor coypus) y el cuis (Cavia aperea) (SPANP 1997; Parera 2002). Diferentes especies de cérvidos habitaban históricamente esta zona, entre ellas puede mencionarse al ciervo de los pantanos (Blastocerus dichotomus), venado de las pampas (Ozotoceros bezoarticus) y la corzuela colorada (Mazama guazoubira) que es la única que en la actualidad se halla en la región (Dellafiore y Maceira 1998).

La avifauna es extraordinariamente rica, excediendo la variedad de aves las 330 especies. Entre ellas se pueden mencionar a las familias Anatidae (patos), Ardeidae (garzas y mirasoles), 
Rallidae (gallaretas, pollas, burritos), Scolopacidae (becasas, playeros), Ciconidae (cigüeñas), Threskiornithidae (bandurrias y cuervillos) (De la Peña 1976; SPANP 1997).

En cuanto a los cérvidos, cabe señalar que Blastocerus dichotomus (ciervo de los pantanos) es el mamífero que posee mayor porte, pudiendo llegar a pesar un macho adulto hasta 150 $\mathrm{kg}$, mientras que las hembras son más pequeñas y su peso oscila entre los 90 y 100kg. En general esta especie posee hábitos solitarios y habita en lugares que se encuentran próximos a lagunas, ríos y en zonas anegadas con pastos altos que le proporcionan alimento y cobertura de los predadores (Pinder y Grosse 1991). El otro ungulado que le sigue en importancia respecto de la talla es Ozotoceros bezoarticus (venado de las pampas), aunque con un tamaño mucho menor que aquel, ya que su peso oscila entre los 25 y los $40 \mathrm{~kg}$. Habita en ambientes abiertos aunque también puede hallarse en sectores cerrados y boscosos (Reig et al. 1983). Por último el cérvido más pequeño que habitaba esta zona y que aún se halla presente en la actualidad, es Mazama gouazoubira, cuyo peso promedio se encuentra entre los 20 y $30 \mathrm{~kg}$ (Reig et al. 1985).

Por otro lado hay dos roedores que son de suma importancia por su disponibilidad, abundancia y fácil captura. El de mayor tamaño es Hydrochoerus hydrochaeris (carpincho), que es el roedor más grande del mundo, ya que los ejemplares adultos pesan más de $50 \mathrm{~kg}$ pudiendo alcanzar los $90 \mathrm{~kg}$. El carpincho al igual que Myocastor coypus (coipo) está adaptado al medio acuático y se alimenta de las gramíneas ribereñas. Habita zonas cercanas al agua provistas de vegetación capaz de proporcionarle alimento y también refugio, ya que esta especie no construye albergues sino que aprovecha los refugios naturales (Cabal et al. 1983). El carpincho posee hábitos gregarios, viviendo en manadas sedentarias que varían en su tamaño (de quince a treinta individuos) según las estaciones del año.

El otro roedor de importancia es Myocastor coypus (coipo) el cual posee un tamaño mediano, (que alcanza un peso medio de $7 \mathrm{~kg}$ ) y que se caracteriza por poseer hábitos acuáticos (Olrog y Lucero 1981). El coipo es un excelente nadador y se alimenta de raíces y otras materias de origen vegetal. Este roedor construye plataformas con materiales vegetales cerca del agua y cuevas como refugio. Estas últimas pueden estar conformadas por un túnel sencillo o por un sistema complejo de pasadizos que se extienden unos $15 \mathrm{~m}$ y presentan cámaras donde arman sus nidos (Ferrari 1983).

Por último Cavia aperea es uno de los roedores más pequeños que habitan la zona y que fue identificado, como los taxa anteriormente mencionados, también en BP y CA. Su peso promedio es de $400 \mathrm{gr}$, son animales gregarios, de hábitos diurnos y no realizan cuevas, aunque pueden ocupar las de otros animales (Pardiñas 1999).

Otros taxa característicos de la zona son los dasipódidos, cuyo tamaño es variable según las especies y el género al cual pertenezcan (los representantes más grandes llegan a pesar unos $50 \mathrm{~kg}$ ), pudiendo decirse en términos generales que el promedio es de $6 \mathrm{~kg}$. Dos especies pueden hallarse en la actualidad en la zona: Chaetophractus villosus (peludo) y Dasypus hybridus (mulita). La primera es una especie eurioica con una distribución que abarca gran parte de Argentina, mientras que Dasypus hybridus es una especie de estirpe brasílica que habita los climas cálidos-húmedos del sur de Brasil y hasta el sur de la provincia de Buenos Aires en Argentina. 
La proximidad del sitio a diferentes fuentes de agua, tales como -en orden de proximidad-: la laguna Paiva, el arroyo Palometa, río Paraná Miní y más alejado el río Paraná, hace esperable que en el conjunto arqueofaunístico se identifiquen distintas especies de peces. Entre las más representativas de la zona pueden mencionarse el armado (Pterodoras granulosus), surubí (Pseudoplatystoma coruscans), el manguruyú (Paulicea lutkewi), el patí (Luciopimeledus patî), el dorado (Saliminus maxillosus), la raya de río (Potamotrygonidae sp.), el pacucito (Metynnis maculatus) y las palometas (Serrasalums sp.) (Ringuelet 2004). Los movimientos de estas especies están vinculados al ciclo hidrológico del río Paraná, con lo cual en los períodos de crecientes se produce un activo intercambio de peces con las cuencas leníticas de zonas aledañas. Así ejemplares jóvenes y de mediana talla de diversas especies, más aquellos que habían quedado encerrados en el ciclo anterior y han logrado reproducirse, abandonan estos cuerpos de agua e ingresan a los arroyos o ríos adyacentes (Bonetto et al. 1969). De esta forma, si bien los peces están disponibles anualmente, hay períodos estacionales más óptimos que otros para su explotación.

\section{Descripción del sitio y procedencia de la muestra analizada}

Campo Binaghi se localiza en un campo que actualmente se halla sembrado con algodón y que se encuentra próximo (a unos cien metros) de la laguna Paiva, la cual constituye una fuente de agua permanente. El terreno posee forma de lomada y en su punto más alto alcanza una altitud de $48 \mathrm{msnm}$. A la llegada al sitio se observó que había diseminado por todo el terreno abundante material arqueológico, fundamentalmente fragmentos cerámicos, pero también restos de valvas de moluscos y óseos faunísticos. Probablemente la presencia de estos materiales en superficie se deba a la acción del arado, que habría removido las primeras capas estratigráficas, haciendo que el mismo quede expuesto y alterando su posición original hasta unos $30 \mathrm{~cm}$ de profundidad (que es hasta donde se registraron las concentraciones de material). Por tal motivo se considera que los materiales en superficie serían parte del mismo componente que se halla en estratigrafía.

Los restos arqueofaunísticos recolectados en superficie, corresponden en un $87 \%$ a astillas de huesos largos indeterminados, un $9 \%$ a fragmentos de dientes de artiodactyla y un $4 \%$ a elementos a Blastocerus dichotomus (fragmentos de metapodio, metacarpo, falange y de radio) y a Myocastor coypus (un fragmento de hemimandíbula). El radio de la primer especie mencionada fue hallado próximo al PS2 y presenta huellas de procesamiento (lascado y huellas de corte). Debido a que en los demás sitios a comparar no se consideraron los materiales de superficie, tampoco serán tenidos en cuenta para este análisis los de $\mathrm{CB}$, más allá que, como ya se mencionó, sean parte del mismo conjunto que se halla en estratigrafía. Para su recolección, y la de los demás materiales, se trazaron ocho transectas, (en sentido E-O) de $20 \mathrm{~m}$ de largo por 1,5 $\mathrm{m}$ de ancho, y separadas entre sí por la misma distancia (1,5 $\mathrm{m})$. En aquellos sectores en donde se localizaron las mayores concentraciones de restos arqueológicos (fundamentalmente cerámico) se realizaron dos pozos de sondeos (PS1 y 2) de 0,50 × 0,50 m; hallándose material arqueofaunístico sólo en el primero.

El sondeo 1 se ubicó en la parte más alta del sitio $(48 \mathrm{msnm})$ y se excavó hasta los $0,40 \mathrm{~m}$ de profundidad, registrándose a los 0,30 m una importante concentración de materiales (fundamentalmente arqueofaunísticos) contenidos en un horizonte A con un sedimento negro y otro marrón claro más arcilloso, en el cual los materiales disminuían su presencia. 
El sondeo 2, localizado aproximadamente a unos $15 \mathrm{~m}$ al sur y $30 \mathrm{~m}$ al oeste del primero, se posicionó en un sector más bajo del sitio (a una altitud de $33 \mathrm{msnm}$ ). Se excavó hasta los $0,50 \mathrm{~m}$ de profundidad y la concentración de materiales, particularmente fragmentos cerámicos, se registró en los primeros 0,20 m. En este pozo, a diferencia del PS1, no se recuperaron restos arqueofaunísticos y los materiales cerámicos al igual que en el pozo anterior, se hallaban contenidos en un sedimento negro que cambiaba su composición hacia uno rojizo y más arcilloso a los $30 \mathrm{~cm}$.

\section{Metodología}

En lo que respecta a la metodología del análisis arqueofaunístico, la identificación anatómica y taxonómica se realizó utilizando los criterios de la sistemática biológica, tratando de alcanzar el nivel taxonómico y anatómico más preciso (Salemme et al. 1988). En aquellos casos que por el grado de astillamiento y/o conservación de los materiales no fue factible asignar a los especímenes a ninguna categoría taxonómica (i.e. Familia, Orden, Clase) fueron considerados como fragmentos indeterminados -indet-.

Para estimar la abundancia y diversidad taxonómica se utilizaron las medidas convencionales de NISP y MNI (Grayson 1984; Lyman 1994; Mengoni Goñalons 1988), mientras que el cálculo del NME se realizó mediante el establecimiento de zonas diagnósticas (Mengoni Goñalons 1999). Para el caso de las presas pequeñas que se hayan representadas en el registro (eg. coipo y cavia), éste fue recompuesto teniendo en cuenta sólo tres zonas diagnósticas para cada elemento. La decisión de dividirlos en sólo tres porciones se debió a que los huesos largos de estos taxa son pequeños, no superando los $15 \mathrm{~cm}$ de largo.

El índice de fragmentación se estimó a partir de la relación NISP/MNE (Lyman 1994). Debido a que gran cantidad de huesos se encontraron muy fragmentados se establecieron tres categoría de pertenencia: Mammalia grande (>50kg, ie. Blastocerus dichotomus); Mammalia mediano (<50kg y hasta $10 \mathrm{~kg}$; ie. Ozotoceros bezoarticus, Mazama gouazoubira y Aguará guazú) y Mammalia pequeño ( $<10 \mathrm{~kg}$, ie. Myocastor coypus, Cavia aperea y dasipódidos). Hydrochoerus hydrochaeris (carpincho) se encontraría en la segunda categoría, pero debido a que no se identificó ningún elemento en el conjunto, se descarta por el momento que los restos puedan pertenecer a este taxón.

La diferenciación interespecífica dentro de la Familia Cervidae, debido a las similitudes morfológicas existentes entre las especies, se realizó mediante mediciones osteométricas de las falanges para poder distinguir entre O. bezoarticus y Mazama sp.; aunque en muchos casos debido al estado fragmentario, los especímenes sólo pudieron ser asignados a nivel de Familia. Por otro lado, la determinación etaria de Blastocerus dichotomus se realizó siguiendo el patrón de fusión ósea de Loponte (2004).

La determinación etaria de Myocastor coypus, debido a que la muestra no cuenta con mandíbulas completas o fragmentos de molares in situ no pudo realizarse, ya que ésta se basa en los criterios de erupción de molares y en el desgaste dentario de los mismos (Rusconi 1930).

Por otra parte, los dasipódidos se encontraron representados por placas dérmicas correspondientes a los caparazones, que si bien son un elemento diagnósticos para determinar Género-especie (Vizcaíno y Bargo 1993), cuando se encuentran fragmentados 
ésta se dificulta, dada la similitud morfológica de las mismas en las distintas especies que podrían encontrarse presentes en el registro arqueológico de CB. Por esto, cuando no se pudo establecer con certeza el nivel específico se las clasificó a nivel de familia (Dasypodidae).

En cuanto a los Peces se consideraron como vértebras completas a aquellas que poseían más del 50\% del cuerpo (Gifford-González et. al. 1999, Musali 2005). Cuando los elementos no pudieron ser identificados a niveles taxonómicos de mayor resolución, los mismos fueron referidos a niveles más generales (Familia, Orden, Clase).

También se relevó la incidencia de agentes tafonómicos en la muestra, tales como: la presencia de meteorización (según los estadios establecidos por Behrensmeyer 1978 para mamíferos grandes-medianos y Andrews 1990 para los pequeños), manchas de manganeso, la acción de roedores y de raíces (Lyman 1994; Wood y Johnson 1978). Respecto de las modificaciones antrópicas que sufren los especímenes producto de su procesamiento fueron relevadas las huellas de corte y los negativos de impacto (Mengoni Goñalons 1999).

\section{Análisis del conjunto}

El conjunto arqueofaunístico de CB está compuesto por 565 especímenes que provienen de la excavación del PS1. Sólo un 6,5\% no pudo ser identificado específicamente. En el resto de la muestra predominan los mamíferos respecto de las demás clases identificadas (eg. Peces, Aves) (Tabla 1).

En cuanto a la clase Ave, los especímenes representados corresponden a dos fragmentos de cilindros que se hallaron en una posición más superficial (a los $0,10 \mathrm{~m}$ ) que la del resto

\begin{tabular}{|c|c|c|}
\hline Taxa & NISP & NISP $\%$ \\
\hline Mammalia & 449 & 79,4 \\
\hline Peces & 48 & 8,4 \\
\hline Reptilia & 17 & 3 \\
\hline Malacológico & 12 & 2,1 \\
\hline Ave & 2 & 0,3 \\
\hline Indet. & 37 & 6,5 \\
\hline TOTAL & $\mathbf{5 6 5}$ & $\mathbf{1 0 0}$ \\
\hline
\end{tabular}

Tabla 1. NISP y \% de NISP por Clases y según los diferentes grados de resolución taxonómica del sitio CB. 
del conjunto, presentando además una coloración blancuzca, lo cual podría indicar que corresponden a restos actuales. No ocurre lo mismo con las falanges de Ovis aries, las cuales fueron halladas a mayor profundidad $(0,25 \mathrm{~m})$ y con una coloración similar a la del resto de los materiales. Sin embargo, hasta que no se cuente con fechados del sitio, las mismas serán consideradas como material intrusivo. Esto se debe a la posible migración vertical que pudo haberse producido en el sitio, sobre todo tratándose de elementos que poseen características que los hacen factibles de introducirse en la matriz sedimentaria (Borrero 1990), y que se hallan en un contexto en donde la remoción de los primeros centímetros del terreno es permanente.

La Familia Cervidae es una de las que se encuentra más representada (ver Tabla 1), correspondiendo ocho falanges y un fragmento de metapodio a B. dichotomus. Con mayor abundancia se encuentra $O$. bezoarticus, representado en su totalidad por elementos correspondientes al esqueleto apendicular (ver Tabla 2). Cabe señalar que en este caso no se descarta la posible pertenencia de algunos elementos a Mazama gouazoubira, aunque la distinción osteométrica entre ambas especies se encuentra en un estado inicial y se dificulta en el presente caso el poder realizarla debido al estado fragmentario de la muestra (ver Acosta et al. 2011).

\begin{tabular}{|l|c|c|c|c|}
\hline O.bezoarticus & MNE & MAU & MAU $\%$ & MNI \\
\hline $1^{\circ}$ y $2^{\circ}$ falange & 7 & 0,4 & 40 & - \\
\hline Metapodio diaf. & 2 & 0,5 & 50 & 1 \\
\hline Húmero ds. & 1 & 0,5 & 50 & 1 \\
\hline Tibia px. & 2 & 1 & 100 & 2 \\
\hline Radio px. & 1 & 0,5 & 50 & 1 \\
\hline Astrágalo & 2 & 1 & 100 & 2 \\
\hline Escafoides & 1 & 0,5 & 50 & 1 \\
\hline Maleolar & 1 & 0,5 & 50 & 1 \\
\hline Molar & 1 & - & - & 1 \\
\hline TOTAL & 18 & & & \\
\hline
\end{tabular}

Tabla 2. Medidas de abundancia anatómica de O. bezoarticus. 
Dentro de la categoría de Artiodactyla se encuentran en mayor porcentaje, diáfisis de huesos largos indeterminados $(\mathrm{N}=18)$, fragmentos de costilla $(\mathrm{N}=3)$, diáfisis de metapodios $(\mathrm{N}=3)$, fragmentos de molares $(\mathrm{N}=10)$, tres bulas timpánicas, un fragmento de fémur, uno de tibia, escápula, vértebra y dos fragmentos de cúbito y de húmero. Debido a las similitudes específicas no pudieron ser asignados a alguno de los tres taxa posibles, aunque cabe señalar que estos elementos indican secciones del esqueleto que se hallan ausentes entre los especímenes identificados a nivel específico, como el cráneo y otras partes de la sección axial. El índice de fragmentación para Mammalia grande es elevado, alcanzando un 4,3.

Otra Familia muy representada es Rodentia (NISP\% 5,7), en la cual se identificaron a su vez, dos especies: $M$. coypus y C. aperea (Tabla 3). La primera de éstas posee un MNI de 1 mientras que la segunda de 3 , obtenido de las hemimandíbulas y húmero respectivamente. El coipo posee elementos que corresponden tanto al esqueleto axial como al apendicular y lo mismo ocurre con el cuis lo cual hace pensar en el ingreso sin un trozamiento previo de estas presas.

\begin{tabular}{|c|c|c|c|c|c|c|}
\hline & \multicolumn{2}{|c|}{ Myocastor coypus } & \multicolumn{3}{c|}{ Cavia aperea } \\
\hline & NISP & MAU & MAU\% & NISP & MAU & MAU\% \\
\hline Cúbito & 1 & 0,5 & 50 & - & - & - \\
\hline Cráneo & - & - & - & 4 & - & - \\
\hline Carpiano & 1 & - & - & - & - & - \\
\hline Autopodio & 1 & - & - & - & - & - \\
\hline Mandibula & 1 & 0,5 & 50 & 5 & 2,5 & 83,3 \\
\hline Húmero & - & - & - & 6 & 3 & 100 \\
\hline Fémur & 2 & 1 & 100 & 4 & 2 & 66,6 \\
\hline Tibia & - & - & - & 1 & 0,5 & 16,6 \\
\hline Escápula & - & - & - & 1 & 0,5 & 16,6 \\
\hline Cadera & 2 & 1 & 100 & 1 & 0,5 & 16,6 \\
\hline Vértebra & 4 & - & - & 1 & - & - \\
\hline V. caudal & 2 & - & - & - & - & - \\
\hline Molar & 1 & - & - & 1 & - & - \\
\hline Incisivo & 1 & 0,25 & 25 & 2 & 0,5 & 16,6 \\
\hline $4^{\circ}$ falange & 1 & - & - & - & - & \\
\hline $2^{\circ}$ falange & 1 & - & - & - & - & - \\
\hline $1^{\circ}$ falange & 1 & - & - & - & - & \\
\hline TOTAL & 19 & & & 26 & & \\
\hline
\end{tabular}

Tabla 3. Medidas de abundancia anatómica de Myocastor coypus y Cavia aparea. 
El índice de fragmentación para el orden de los roedores, si se considera dentro de esta categoría a los especímenes correspondientes a Mammalia pequeños, es de 3,9; este valor no dista demasiado del de los mamíferos de mayor tamaño, como los cérvidos. Esto sugiere que el conjunto habría sufrido una alta fragmentación, ya sea por la incidencia de agentes antrópicos, naturales o de ambos.

En el caso de C. aperea la mayor proporción de especímenes fusionados y la presencia de todos los molares emergidos indican que se habría preferido la utilización de individuos sub adultos-adultos, por sobre los juveniles.

En cuanto a la familia Dasypodidae dos especies fueron identificadas por la presencia de placas dérmicas, las cuales, si bien son diagnósticas respecto a la determinación específica, no lo son respecto al MNI, debido a la gran cantidad que poseen por individuo. D. hybridus es la más representada $(\mathrm{N}=7)$, siguiéndole $C h$. villosus con seis elementos. La primer especie mencionada es típica del ambiente en el cual se emplaza el sitio, mientras que la segunda es una especie eurioica, con lo cual es probable que se hallen en este tipo de registros.

Otra de las especies que se encuentra representadas es Tupinambis merinae, con un NISP de 10 y un MNI de 1. Los elementos corresponden a vértebras y hemimandíbula, no identificándose ninguno correspondiente al esqueleto apendicular.

Por último, también dentro de la clase Mammalia, se registraron tres elementos pertenecientes a Chrysocyon brachyurus (aguará guazú): dos fragmentos de mandíbula y una falange. Es posible que algún otro espécimen perteneciente a Mammalia mediano no haya podido ser identificado a este nivel y pertenezca a este taxón.

Los restos asignados a la Clase Peces no pudieron ser determinados a nivel específico debido al grado de fragmentación. Fueron recuperados elementos correspondientes a todo el esqueleto (Tabla 4) y los mismos corresponderían a presas pequeñas, menores a 800 grs. Esto se infiere del tamaño de las vértebras, ya que las mismas muestran una buena correlación con el tamaño y peso de los peces (Desse-Berset 1984).

\begin{tabular}{|c|c|}
\hline Actinopterigii & MNE \\
\hline vértebras & 31 \\
\hline cráneo+neurocráneo & 9 \\
\hline costillas & 2 \\
\hline espinas pectorales & 4 \\
\hline dientes & 2 \\
\hline Total & 48 \\
\hline
\end{tabular}

Tabla 4. MNE de Clase Peces 


\section{Consideraciones tafonómicas}

A pesar que el sitio se encuentra en un área modificada antrópicamente por la acción del arado, sólo se observaron indicios de meteorización en un $10 \%$ de la muestra, y los mismos se corresponden con estadíos bajos, no registrándose ningún caso mayor a 3 según la escala de Behernsmeyer (1978). Entre los mamíferos pequeños como C. aperea, los estadios registrados también fueron bajos agrupándose entre 0 y 1 (Andrews 1990). Esto le confiere a la muestra un buen estado de preservación que permite el relevamiento de las modificaciones en el tejido cortical de los especímenes.

Las raíces serían el factor que mayor influyó en la modificación de las superficies óseas, encontrándose presente el patrón dendrítico (en este caso de un color marrón claro) en un $33 \%$ de los especímenes. Este patrón respondería a la vegetación arbustiva que cubre el sitio plantada en la actualidad.

Las manchas de manganeso se hayan presentes en un $8 \%$ y esto estaría indicando que en el sitio se habrían producido una alternancia entre ciclos de reducción-oxidación, en donde el componente de arcilla o carbonato del suelo impide la rápida filtración del agua (Courty et al. 1989).

Los roedores tuvieron una incidencia baja en el conjunto, registrándose las marcas en sólo dos fragmentos de diáfisis de mamífero, mientras que la de los carnívoros es nula. Las termoalteraciones se observaron en un $9 \%(\mathrm{~N}=51)$ y entre las especies afectadas se hallan $B$. dichotomus (2 falanges), O. bezoarticus (1 astrágalo), M. coypus (cadera y fémur) y Ch. villosus (1 placa dérmica). Sin embargo, la mayor cantidad de especímenes corresponden a fragmentos de huesos largos de Mammalia grande y mediana.

Al momento de determinar el uso de alguno de los recursos por parte de las poblaciones humanas, la muestra analizada presenta principalmente dos desventajas: por un lado, posee una baja cantidad de especímenes representados por taxón y por otro, un alto grado de fragmentación de los mismos. Esto dificulta afirmar que efectivamente hubo un aprovechamiento antrópico de esas especies, ya que las inferencias se realizan principalmente en base a dos propiedades observables físicamente en los especímenes faunísticos: la presencia de huellas de corte y de fracturas intencionales.

En el conjunto de CB se observaron huellas de corte (Binford 1981; Mengoni Goñalons 1999 ) en un $2,3 \%$ de los especimenes $(n=13)$. Las mismas se hallan en fragmentos de huesos largos de mammalia, en un fémur de coipo y en una falange de aguará guazú. Las fracturas intencionales se registraron en menor proporción que las huellas, sólo en un 1\% (N=6) de todo el conjunto. Estas se hallan en elementos asignados a la familia Cervidae, son de tipo longitudinal, espiral, y se registraron en fragmentos de huesos largos (tibia y metapodio) y falanges.

No obstante, las bajas proporciones de modificaciones antrópicas no deben ser consideradas como una evidencia negativa, ya que para un conjunto de tales características es esperable. Hay evidencias de sitios con NISP más abundantes que demuestran una baja presencia de huellas (ver Santiago 2001; Escudero y Feuillet Terzaghi 2007: González 2005) y en algunos casos nula (Acosta y Pafundi 2005). 
Debe tenerse en cuenta que las huellas corresponden a un epifenómeno, es decir a un accidente durante el procesamiento de las presas, por lo tanto su presencia y frecuencia dependerá de diversas variables, tales como la actividad que se esté realizando (desarticulación, cuereo, etc), el tamaño de la presa, la morfología del hueso, el instrumental empleado, la intensidad del procesamiento (total o parcial), etc. (Lyman 2005).

En cuanto a especies pequeñas como el coipo y cavia, su tamaño es uno de los factores que pueden estar incidiendo en que las huellas se hallen en muy baja proporción (en el primero de los casos, sólo una en un fémur y en el segundo, ninguna). Estas presas podrían haber ingresado enteras al sitio y por lo tanto, habrían requerido un procesamiento menos intensivo. Otro factor refiere al tipo de partes anatómicas representadas, ya que según un estudio comparativo realizado para coipo en el sector de la cuenca inferior del río Paraná, éstas son recurrentes en huesos largos (húmero, cúbito, fémur y tibia) y en ciertas zonas de la hemimandíbula (Sartori y Colasurdo 2012). Cabe señalar que justamente en CB estos elementos se hallan con una baja representación y una alta fragmentación.

Además, los indicadores que apuntan a la utilización humana de Myocastor coypus (más que a su ingreso natural al registro) son: 1) hasta el momento no se registraron las cuevas típicas que realizan estos mamíferos para sus madrigueras, 2) no hay marcas de estos roedores en los materiales arqueológicos analizados hasta el momento, 3) se observaron huellas de procesamiento y 4) los restos carecen de marcas de carnívoros y no serían una presa apta para las aves estrigiformes (Acosta y Pafundi 2005).

La presencia de alteraciones térmicas, también puede ser considerada como indicador de actividad antrópica. El hecho de que una placa dérmica de Chaetoprhactus villosus se halle quemada, podría atribuirse a la cocción entera de esta pieza mediante el asado (Feuillet Terzaghi 2002); no obstante, las termoalteraciones también pueden deberse al descarte de los especímenes faunísticos en un fogón, ya sea para mantener limpio el lugar o para su utilización como combustible.

Respecto de la clase Peces, el predominio de vértebras por sobre los demás elementos del esqueleto indicaría que el ingreso al registro de estos taxa responde a factores antrópicos. Esto se infiere porque en las acumulaciones de peces que son naturales suelen encontrarse sobre-representadas las partes con mayor densidad ósea (eg. cráneo) (Zohar et al. 2001; Gifford-González et al. 1999), llegando a predominar hasta en un 70\% de la muestra (según Martínez et al. 2004).

En síntesis, se considera que la mayoría de las especies representadas en el sitio habrían ingresado al mismo principalmente producto de la actividad antrópica. No obstante lo expuesto, es posible que algunos ejemplares de reptiles y de cavia hayan ingresado al sitio producto de agentes naturales. Este ingreso podría estar dado por cuestiones etológicas de cada especie, a que su tamaño los hace aptos para ser consumidos por diferentes depredadores, y a que no poseen huellas antrópicas.

Por último, también se encontraron algunas evidencias de la utilización ósea como materia prima para la producción de instrumentos. En tal sentido fueron hallados un ápice que podría corresponder a una punta acanalada o semiacanalada -ahuecada o con epífisis -(ver Pérez Jimeno 2007; Buc y Pérez Jimeno 2010) y un fragmento de diáfisis de metapodio de 
ciervo con desgaste intencional (seguramente para su aguzamiento). También se recuperó una diáfisis de mamífero con una fractura recta probablemente generada posteriormente al marcado perimetral, característico de los desechos de tecnofacturas hallados en los otros registros analizados (Pérez Jimeno 2007). Esto permite suponer que la alta fragmentación de los materiales pudo deberse, en parte, a la confección de instrumentos y no sólo a la acción de agentes tafonómicos (ver Pérez Jimeno 2007).

\section{Discusión}

Hasta el momento las evidencias existentes en la llanura aluvial del Paraná Medio reflejan que la misma fue ocupada hacia el Holoceno Tardío, con posterioridad a 1500 años AP, cuando se produjo el último cambio climático, conocido como "Máximo Medieval". Allí fue cuando se establecieron las condiciones de temperatura y humedad actuales, y el valle del Paraná adquirió la fisonomía que hoy lo caracteriza para la ocupación humana (Iriondo 1991,1999; Cioccale 2000).

De acuerdo a las características de los registros zooarqueológicos de los sitios $\mathrm{BP}$ y CA, su posición estratigráfica (Pérez Jimeno 2007) y los fechados radiocarbónicos de este último (Tabla 5), es posible situarlos cronológicamente en dicho período (Pérez Jimeno 2004, 2007).

\begin{tabular}{|c|c|c|c|c|}
\hline Sitio & 14C a. AP \pm 1 s & Edad Calibrada & Cód. Lab. & Referencia \\
\hline \multirow{2}{*}{$\begin{array}{c}\text { Cerro } \\
\text { Aguará }\end{array}$} & $530 \pm 70$ & $1327-1441$ años cal AD & \multirow{2}{*}{ LP-1431 } & \\
\cline { 2 - 3 } & & $509-623$ años cal A.P. & & $\begin{array}{c}\text { Pérez } \\
\text { Jimeno } \\
\end{array}$ \\
\cline { 2 - 3 } & $895 \pm 60$ & $1039-1224$ AD & \multirow{2}{*}{ LP-1395 } & \\
\cline { 3 - 3 } & & $726-911$ años cal A.P. & & \\
\hline
\end{tabular}

Tabla 5. Fechados radiocarbónicos del sitio CA.

Si bien los tres sitios aquí considerados se encuentran dentro de la llanura aluvial del Paraná, CA y BP están emplazados respectivamente sobre un "cerrito" y un albardón (ver Pérez Jimeno 2007) en el área baja de Florencia (Brandolín 1984). Actualmente los terrenos son utilizados para el pastoreo de ganado vacuno y a diferencia de CB no son aptos para el cultivo. Barrancas del Paranacito fue excavado por el equipo de Lafón durante 1971- 1972, mientras que Cerro Aguará por el equipo de una de las autoras de este manuscrito (Pérez Jimeno 2007). Ambos son considerados sitios de actividades múltiples; en tanto que BP también fue utilizado como área de enterratorios.

De acuerdo a los estudios zooarqueológicos realizados, los grupos que habitaron dichos sitios habrían tenido una subsistencia basada principalmente en la caza, pesca y recolección de moluscos. Hasta el momento no se cuenta con evidencias del aprovechamiento de recursos vegetales propios del área, aunque esta posibilidad no se descarta hasta que no se profundicen los análisis. Las estrategias utilizadas en la captura y procesamiento de los recursos se asemejan, y ambos conjuntos demuestran un amplio desarrollo de la tecnología ósea y cerámica, mientras que los materiales líticos están totalmente ausentes. 
No obstante las diferencias significativas respecto de las superficies excavadas en cada uno de los sitios $(C A=5,68 \mathrm{~m} 3 ; B P=56,4 \mathrm{~m} 3 ; \mathrm{CB}=0,125 \mathrm{~m} 3)$, se realiza aquí un análisis comparativo preliminar, considerando la presencia/ausencia de especies en los distintos conjuntos (Tabla 6).

\begin{tabular}{|c|c|c|c|c|c|c|c|}
\hline \multirow{2}{*}{$\begin{array}{c}\text { Taxa } \\
\text { Mammalia indeterminado }\end{array}$} & \multirow[t]{2}{*}{ nombre vulgar } & \multicolumn{2}{|c|}{$\begin{array}{c}\text { CA } \\
\% \text { NISP } \\
N=32997\end{array}$} & \multicolumn{2}{|c|}{$\begin{array}{c}\text { BP } \\
\% \text { NISP } \\
\text { N=4758 }\end{array}$} & \multicolumn{2}{|c|}{$\begin{array}{c}\mathrm{CB}^{*} \\
\text { NISP\% } \\
\mathrm{N}=565\end{array}$} \\
\hline & & $x$ & 0,8 & $x$ & 32,0 & $\mathrm{x}$ & 49 \\
\hline Artiodactyla & & - & - & - & - & $x$ & 1,4 \\
\hline Cervidae & & $x$ & 0,04 & $x$ & 0,7 & $x$ & 6,7 \\
\hline Blastocerus dichotomus & ciervo de los pantanos & $x$ & 0,8 & $x$ & 4,5 & $x$ & 1,5 \\
\hline Ozotoceros bezoarticus & venado de las pampas & $x$ & 1,9 & $\mathrm{x}$ & 4,0 & $\mathrm{x}$ & 3,1 \\
\hline Bos Taurus & vaca & $x$ & 0,06 & $\mathrm{x}$ & 1,6 & - & - \\
\hline Ovis aries & oveja & - & - & - & - & $x$ & 0,3 \\
\hline Rodentia & & $x$ & 0,03 & - & - & $\mathrm{x}$ & 5,6 \\
\hline Hydrochoerus hydrochaeris & carpincho & $x$ & 1,07 & $x$ & 1,7 & - & - \\
\hline Myocastor coypus & coipo & $x$ & 0,6 & $\mathrm{x}$ & 2,7 & $\mathrm{x}$ & 3,3 \\
\hline Cavia aperea & cuis & $x$ & 6,2 & $\mathrm{x}$ & 20,7 & $\mathrm{x}$ & 4,6 \\
\hline Holochilus chacarius & rata colorada & $x$ & 0,03 & - & - & - & - \\
\hline Cricetidae sp. & & - & - & - & - & $\mathrm{x}$ & 0,3 \\
\hline Dasipódidos & Mulita, peludo & - & - & - & - & $\mathrm{x}$ & 1,6 \\
\hline Chrysocyon brachyurus & aguará guazú & $x$ & 0,003 & - & - & $\mathrm{x}$ & 0,5 \\
\hline Carnívora & & $x$ & 0,003 & - & - & - & - \\
\hline Didelphis sp. & comadreja & $x$ & 0,003 & $\mathrm{x}$ & 0,2 & - & - \\
\hline Peces & & $x$ & 88,4 & $x$ & 18,7 & $\mathrm{x}$ & 8,5 \\
\hline Reptilia & & $x$ & 0,11 & $\mathrm{x}$ & 0,79 & $x$ & 1,2 \\
\hline Caiman sp. & yacaré & $x$ & 0,4 & $x$ & 3,32 & - & - \\
\hline Pleurodira & tortuga & $x$ & 0,1 & - & - & - & - \\
\hline Phrynops hilarii & tortuga de agua & $x$ & 0,1 & $\mathrm{x}$ & 0,97 & - & - \\
\hline Tupinambis sp. & iguana & $x$ & 0,1 & $\mathrm{x}$ & 0,69 & - & 1,7 \\
\hline Aves & & $x$ & 0,4 & $\mathrm{x}$ & 9 & $\mathrm{x}$ & - \\
\hline Rhea americana & ñandú & $x$ & 0,009 & & - & - & - \\
\hline
\end{tabular}

Tabla 6. NISP\% de los taxa presentes en CA, BP y CB.

En primera instancia, es necesario destacar que Campo Binaghi refleja la utilización de algunos recursos que no se hayan presentes en los otros registros considerados aquí como lo es la familia Dasipodidae. Su ubicación estratégica, como ya se mencionó, podría explicar dicha presencia. Además, como se refirió, la evidencia de termoalteraciones en uno de estos taxa, permite pensar que posiblemente el peludo y la mulita hayan sido aprovechados como alimento.

Por otro lado, los conjuntos de Campo Binaghi y Barrancas del Paranacito, a diferencia de Cerro Aguará, reflejan una mayor explotación de mamíferos terrestres que de peces. En los tres sitios, predominan los cérvidos y en segundo lugar los roedores. No obstante, en Campo Binaghi no fue identificado ningún espécimen correspondiente a Hydrochoerus hydrochaeris 
(carpincho), mientras que en CA y en BP se encuentra ampliamente representado por especímenes correspondientes a todo el esqueleto y se registraron huellas de procesamiento (Santiago 2004: Pérez Jimeno 2007).

Además, y siempre considerando que las tendencias observadas en la muestra de Campo Binaghi se mantuviesen, se pueden señalar aspectos vinculados a preferencias específicas, en este caso de $O$. bezoarticus por sobre B. dichotomus. Este hecho es esperable, ya que el venado a diferencia del ciervo, habita principalmente ambientes abiertos como en el que se emplaza CB (Reig et al. 1983). No obstante, en los tres casos, estas especies están representadas principalmente por el esqueleto apendicular, lo cual estaría hablando de similitudes en el tratamiento de las carcasas.

Es importante señalar, que en BP y CB los peces están escasamente representados. Si bien estos sitios se encuentran más hacia el oeste que CA y emplazados en áreas más abiertas, ambos sitios se encuentran próximos a diferentes fuentes de agua, lo que les hubiese facilitado el aprovechamiento de diferentes recursos acuáticos. Para BP se propuso como explicación que la escasez podría deberse a que las cabezas y espinas fueron desechadas en otro lugar, por lo que disminuye el NISP recuperado en el sitio; y a que los peces se conservaron mediante secado para un consumo diferido, o bien, procesados como harina (Pérez Jimeno 2007). En cuanto a CB, habrá que ampliar la excavación y profundizar el estudio de este sitio para poder tener mayores precisiones que expliquen esta baja representación, aunque no se descartan por el momento las consideraciones realizadas anteriormente para BP.

Por el contrario, CA cuenta con una amplia representación de peces lo cual permitió afirmar que la pesca fue una actividad importante para los habitantes del sitio. Estos habrían explotado principalmente este recurso en los cuerpos de agua más próximos y no en el río Paraná, prefiriendo la captura en masa mediante el uso de redes (Pérez Jimeno 2007, 2009). El conjunto cuenta con una riqueza específica de 23, constituyéndose de este modo en el sitio con mayor variabilidad dentro de la cuenca del Paraná (ver Musali et al. 2012 en prensa). Este hecho puede explicarse debido al empleo de métodos de grano fino durante la recuperación de los restos, ya que es uno de los pocos conjuntos en donde se utilizó malla de $1 \mathrm{~mm}$ para el cernido (véase Pérez Jimeno 2007). En este sitio más de la mitad de los especimenes identificados corresponden a H. commersonni (vieja del agua), P. maculatus (bagre blanco) y T. galeatus (torito). En términos generales los Siluriformes dominan todos los conjuntos de la zona, y se trata de especies de talla más bien pequeña, que en su mayoría no superarían el kilogramo de peso.

Otra diferencia que es posible señalar, al menos de acuerdo a los datos disponibles hasta el momento, es que las aves en CB están ausentes (ver más arriba Consideraciones tafonómicas). Esto constituye una diferencia con CA y BP, en donde si bien no están altamente representadas respecto de las otras clases ( $0,4 \%$ y $9 \%$ respectivamente), tienen una presencia considerable comparando los registros faunísticos, incluso de otros sectores de la cuenca del Paraná (Acosta et al.2010). Asimismo, su aprovechamiento puede haber sido integral teniendo en cuenta que también fueron utilizados los huesos de éstas como soportes de instrumentos óseos (Pérez Jimeno 2004 y 2007). Se descarta que esta ausencia de aves en el registro pueda deberse a condiciones de preservación, debido a que la muestra posee una historia tafonómica que ha favorecido a la supervivencia de los especímenes de especies pequeñas como roedores y peces, al igual que en los otros sitios considerados (Pérez Jimeno 2007). 
Es notable la ausencia o escasez de Aves en los sitios del humedal, sobre todo, teniendo en cuenta su gran disponibilidad en el ambiente, y que muchas de las especies acuáticas son sumamente predecibles y de fácil captura (Paucke 1942). Además, algunas especies a pesar de las dificultades para su captura, como Rhea americana, propia de áreas abiertas, fueron aprovechadas por distintos grupos humanos en el pasado, tales como los mocovíes (Paucke 1942). Dos opciones habrían para explicar dicha ausencia: a) que se deba a una elección cultural (o a otros factores que son intrínsecos de cada configuración social); o b) debido a cuestiones de sesgo en el muestreo. Se considera que para aseverar cualquiera de estas dos hipótesis deberá realizarse un muestreo más amplio de este sitio.

En relación a los reptiles, se puede señalar que hasta el momento en CB solo está representado Tupinambis merinae, mientras que en CA y BP también se registraron elementos correspondientes a Phrynops hilarii (tortuga de agua) y Caiman sp (yacaré). Asimismo, en CA, se observaron huellas de procesamiento en una vértebra de este último taxón y en una placa propia del caparazón de tortuga (Pérez Jimeno 2007). La presencia de yacaré en estos dos sitios es más esperable que en $\mathrm{CB}$, si se tiene en cuenta que se encuentran en un área donde la vegetación es más abundante y cerrada, siendo más óptima para su hábitat.

Por último, es necesario mencionar que tanto BP como en CA son concheros, formados principalmente por Diplodon sp., producto del consumo humano, además de la utilización de sus valvas como materia prima para hacer cuentas de collar, sin descartar otros posibles usos (ver Pérez Jimeno 2007; 2008); mientras que en CB solo hasta el momento se han encontrado algunos valvas diseminadas en la superficie.

\section{Consideraciones finales}

Si bien se entiende que a futuro debería ampliarse la excavación del sitio CB, para poder hallar una muestra más significativa de restos arqueofaunísticos, este análisis preliminar aportó datos para una zona de la llanura Aluvial del Paraná de la cual no se poseía información hasta el momento.

Asimismo, el conjunto analizado posee particularidades que hacen que se amplíe la perspectiva a nivel regional de este sector, ya que la variabilidad arqueofaunística que presenta permitió observar tanto similitudes como diferencias respecto de otros sitios de la región. A manera de síntesis se puede decir que:

a- Si bien aún no se cuenta con fechados radiocarbónicos para $\mathrm{CB}$, de acuerdo a las características del registro arqueológico y a la información existente hasta la fecha es posible situarlo cronológicamente en el Holoceno tardío.

b- El o los grupos que habitaron $\mathrm{CB}$, habrían sido cazadores-recolectores que explotaban recursos faunísticos principalmente propios de áreas topográficamente más altas y abiertas que los de los sitios BP y CA. Es probable que este espacio haya sido ocupado durante los períodos de inundaciones, en los que estos grupos humanos prefirieron aprovechar mamíferos típicos de este ambiente (como O. bezoarticus, $C$. aperea) y no aquellos que se encuentran estrechamente vinculados a los recursos acuáticos, como el caso de los peces, el coipo y el carpincho. 


\section{Agradecimientos}

En primera instancia queremos agradecer la Comuna de Las Toscas por su apoyo logístico y de gestión para la realización de los trabajos arqueológicos realizados en "Campo Binaghi"; así como también, a las Lic. Ma. Belén Colasurdo y Sandra Escudero, y a los estudiantes Fabricio Coviello y Cecilia Servín por participar en la prospección y excavación del sitio. Asimismo, agradecemos a Anahí Hernández por colaborar en la identificación taxonómica y anatómica de la muestra y al Dr. Daniel Loponte por su colaboración en la determinación de los especímenes pertenecientes a la Familia Cervidae; y a Cecilia Servín por ayudarnos a mejorar la figura referida a la ubicación de los sitios estudiados. Además agradecemos a los evaluadores quienes con su aporte contribuyeron a enriquecer significativamente este trabajo. Por último las autoras queremos aclarar que somos las únicas responsables de lo aquí expuesto.

\section{Bibliografía citada}

Acosta, A. y L. Pafundi

2005 Zooarqueología y Tafonomía de Cavia aperea en el humedal del Paraná inferior. Intersecciones en Antropología 6:59-74.

Acosta, A., Escudero, S., Feuillet Terzaghi M.R, Loponte D. y L. Pérez Jimeno 2010 Conectando registros: variabilidad arqueológcia en la Cuenca del Paraná. En Mamül Mapu: pasado y presente desde la arqueología pampeana, (ed. por M. Berón, L., Bonomo M., Montalvo C., Aranda C. y M. Carrera Aizpitarte), Tomo 2:17-28. Libros del Espinillo, Buenos Aires.

Acosta, A, Mucciolo L., Musali J. y M. Arrizurieta

2011 Avances y problemas relacionados con el estudio del registro arqueofaunístico generado por los grupos cazadores-recolectores del extremo sur de la provincia de Entre Ríos (Humedal del río Paraná Inferior). Avances y persepectivas en la arqueología del Nordeste, (ed. por M.R Feuillet Terzaghi, M.B Colasurdo, J. Sartori y S. Escudero). Servicios Gráficos, Buenos Aires.

Andrews, A. P.

1990 Owls, caves and fossils. University of Chicago Press, Chicago.

Behrensmeyer, A.

1978 Taxomic and ecological information from Bone Weathering. Palaeobiology 4 (2): 150 -162.

Binford, L.

1981 Bones. Ancient men and modern myths. Academic press, Orlando.

Bonetto A., E. Cordiviola de Yuan, C. Pignalberi y O. Oliveros

1969 Ciclos hidrológicos del río Paraná y las poblaciones de peces contenidas en las cuencas temporarias de su valle de inundación. Physis XXIX 78:2213-223.

Borrero, L. A.

1990 The taphonomy of guanaco bones in Tierra del Fuego. Quaternary Research 34: 361-371.

Brandolin, $\mathrm{H}$.

1984 Geografía del Distrito Florencia. MS. 
Buc, N. y L. Pérez Jimeno

2010 Puntas para la comparación. Tecnología ósea en el Paraná medio e inferior. Zooarqueología a principios del siglo XXI: aportes teóricos, metodológicos y casos de estudio, (ed. por M. De Nigris, P. M. Fernández, M. Giardina, A. F. Gil, M. A. Gutiérrez, A. Izeta, G. Neme y H. D. Yacobaccio).

Cabal, G. Reig M. y O. Marchetti

1983. El carpincho. Fauna Argentina nº 2., Centro de Editores de América Latina S.A, Buenos Aires.

Cabrera A. y J. Yepes

1940 Mamíferos Sudamericanos: Vida, costumbres y descripción. Compañía Argentina de Editores, Buenos Aires.

Cioccale, M. A.

1999 Climatic fluctuations in de Central Region of Argentina in de last 1000 years. Quaternary International 62: 35-47.

Courty, M.A, Goldberg, P. y M, Richard

1989 Soils and Micromorphology in Archaeology. Cambridge University .Press, Cambridge.

De la Peña, M.

1976 Aves de la provincia de Santa Fe. Castelvi, Santa Fe.

Dellafiore C. y N. Maceira

1998 Problemas de conservación de los ciervos autóctonos de la Argentina. Mastozoología Neotropical 5(2):137-145.

Desse-Berset, G.

1984 Nouvelle contribution à la diagnose des pièces rachidiennes des poissons. Fish Osteoarchaeology Meeting, CNRS (ed. por G. Desse-Berset). Centre des recherché archéologiques.

Escudero, S. y M.R, Feuillet Terzaghi

2007 El registro arqueofaunístico de Cavia aperea en el sitio Playa Mansa (Provincia de Santa Fe). Implicancias antrópicas y tafonómicas. XXVII Encuentro de Geohistoria Regional del Nordeste, IIGHI, CONICET- UNNE, Asunción.

Feuillet Terzaghi, M.R.

2002 El registro arqueológico de vertebrados del sitio Playa Mansa I (Arroyo Seco, Dpto. Rosario, Pcia. Santa Fe). Aportes y perspectivas. Tesis de licenciatura en Antropología. Departamento de Arqueología. Escuela de Antropología. Facultad de Humanidades y Artes, UNR.

Gifford-González, D., Stewart, K. and N. Rybczynski.

1999 Human activities and site formation at modern lake margin foraging camps in Kenya. Journal of Anthropological Archaeology 18:397-440.

González, M.I.

2005 Arqueología de alfareros, cazadores y pescadores pampeanos. Colección Tesis Doctorales. Sociedad Argentina de Antropología, Buenos Aires. 
Grayson, D.

1984 Quantitative Zooarchaeology. Topics in the Analysis of Archaeological Faunas. Academic Press, Orlando.

Iriondo, $\mathrm{M}$.

1991 El Holoceno en el Litoral. Museo Provincial de Ciencias Naturales "FlorentinoAmeghino" (Nueva Serie) 3 (1): 1-39.

1999 Climatic changes in the South American plains: Records of continent-scale oscillation. Quaternary International 57/58: 93-112.

Lafón, C.R.

1971 Introducción a la arqueología del Nordeste argentino. Relaciones de la Sociedad de Antropológica 5: 119-152.

1972 El replanteo para la arqueología del Noreste argentino. Antiquitas 14: 1-16.

Loponte, D.

2004 Atlas osteológico de Blastocerus dichotomus (ciervo de los pantanos). Los Argonautas, Buenos Aires.

Lyman L.

1994 Vertebrate taphonomy. Cambridge Press, New York.

2005 Analyzing cut marks: lessons from artiodactyl remains in the northwestern United States. Journal of Archaeological Science 32: 1722-1732.

Martínez,G., Zangrando, A.F y L., Stoessel

2005 Sitio El Tigre (Pdo. de Patagones, Pcia. de Buenos Aires, Argentina): evidencias sobre la explotación de peces en el curso inferior del río Colorado e implicaciones para los sistemas de subsistencia. Magallania 33(2):99-114.

Mengoni Goñalons, G.

1988 El estudio de huellas en arqueofauna, una vía para reconstruir situaciones interactivas en contextos arqueológicos. Aspectos teóricos-metodológicos y técnicas de análisis. De procesos, contextos y otros huesos (ed. por A. Haber y N. Ratto), pp: 17-29. Buenos Aires.

1999. Cazadores de guanacos de la estepa patagónica. Sociedad Argentina de Antropología. Buenos Aires, Argentina.

Musali, J.

2005 Ictoarqueología del Delta del río Paraná inferior. El sitio laguna La Bellaca 2 como caso de estudio. Tesis de Licenciatura inédita en Antropología. Departamento de ciencias Antropológicas. Facultad de Filosofía y Letras, Universidad de Buenos Aires.

Musali, J., Feuillet Terzaghi M.R y J. Sartori

2012 Análisis comparativo de conjuntos ictioarqueológicos generados por cazadoresrecolectores durante el Holoceno tardío en la baja Cuenca del Plata (Argentina). Revista Cuadernos, en prensa.

Olrog, C y M. Lucero

1981 Guía de mamíferos argentinos. Ministerio de Educación y Cultura. Fundación Miguel Lillo, San Miguel de Tucumán. 
Pardiñas, U. F.

1999 Tafonomía de microvertebrados en yacimientos arqueológicos de Patagonia (Argentina). Arqueología 9:265-340.

Parera, A

2002 Los mamíferos de la Argentina y la región austral de Sudamérica. El Ateneo, Buenos Aires.

Paucke F.

1942 Hacia allá y para acá (Una entrada entre los indios mocobies, (1749-1767). Coni, TucumánBuenos Aires.

Pérez Jimeno, L. 1996 Análisis arqueofaunístico del Sitio Barrancas del Paranacito. Florencia. Departamento General Obligado. Provincia de Santa Fe. Tesis de Licenciatura inédita. Facultad de Humanidades y Artes. Universidad Nacional de Rosario, Rosario.

2004 Análisis comparativo de dos conjuntos de artefactos óseos procedentes de la llanura aluvial del Paraná y la pampa bonaerense. Aproximaciones Contemporáneas a la Arqueología Pampeana. Perspectivas teóricas, metodológicas, analíticas y casos de estudio (ed. por G. Martínez, M. A. Gutiérrez, R. Curtoni, M. Berón y P. Madrid), pp: 319-334. Facultad de Ciencias Sociales, Buenos Aires.

2005 Análisis Zooarqueológico del Sitio “Barrancas del Paranacito (Florencia, Departamento General Obligado -Provincia de Santa Fe). Revista Arqueología 6: 175-201

2007 Investigaciones arqueológicas en el sector septentrional de la llanura aluvial del Paraná-margen santafesina-: la variabilidad del registro arqueológico. Tesis Doctoral. Facultad de Ciencias Naturales y Museo, Universidad Nacional de la Plata.

2008 Presencia y utilización de moluscos de agua dulce en un sitio del humedal paranaense. Revista de la Escuela de Antropología 14: 25-32.

Pinder, L. y A. P. Grosse

1991 Blastocerus dichotomus. Mammalian Species 380: 1-4.

Reig O., Marchetti B. y N. Sarmiento

1983 El venado de las pamapas. Fauna Argentina n ${ }^{\circ} 33$. Centro de Editores de América Latina S.A, Buenos Aires.

1985 Las corzuelas. Fauna Argentina n ${ }^{\circ}$ 77. Centro de Editores de América Latina S.A, Buenos Aires.

Ringuelet, R.

2004 Zoogeografía y ecología de los peces de aguas continentales de la Argentina y consideraciones sobre las áreas ictiológicas de América del Sur. Ecosur 2 (3): 1-122.

Ruggeroni, D.

1975 Arqueología del Paraná. Yacimiento de Isla del Indio. Museo Municipal de Arqueología $\mathrm{N}^{\circ}$ 2. Municipalidad de Reconquista- Dirección de Cultura, Reconquista- Santa Fe.

Rusconi, C.

1930 Evolución craneodental de la nutria (Myocastor coypus bonariensis) a través de su desarrollo postembrionario. Anales de la Sociedad Científica Argentina 22: 5-31. 
Salemme M., Miotti L. y E. Tonni

1988 La determinación sistemática de los mamíferos en el análisis arqueofaunístico. De procesos, contextos y otros huesos (ed. por A. Haber y N. Ratto), pp. 65-73. Buenos Aires.

Santiago, F.

2004 Los Roedores en el "menu" de los habitantes de Cerro Aguará (Provincia de Santa Fe): su análisis arqueofaunístico. Intersecciones en Antropología 5:3-18.

Sartori, J. y M.B, Colasurdo

2012 La recurrencia del coipo (Myocastor coypus) en los registros arqueofaunísticos de la Cuenca Inferior del río Paraná (Argentina). Arqueología Iberoamericana 13:23-3.

Schmitz P., Ceruti C., González A. R. y A. Rizzo

1972 Investigaciones arqueológicas en la zona de Goya (Corrientes), Argentina. Dédalo. Órgano oficial do Museu de Arqueologia e Enologia da Universidade de Sao Paulo, Brasil.

SPANP (Sistema provincial de Áreas Naturales Protegidas de Santa Fe). 1997 Sistema Provincial de Áreas Naturales Protegidas. Gobierno de la Provincia de Santa Fe, Administración de Parques Nacionales. Asociación Cooperadora de la E.Z.E. Talleres Gráficos de Imprenta Ciscato. Santa Fe, Argentina.

Stewart K. y D. Giffrod-González

1994 An Ethnoarchaeological Contribution to Identifying Hominid Fish Processing Sites. Journal of Archaeological Science 21:37-248.

Vizcaíno, S.F y M.S, Bargo

1993 Los armadillos (Mammalia Dasypodidae) de La Toma (Partido de Coronel Pringles) y otros sitios arqueológicos de la Provincia de Buenos Aires. Consideraciones paleoambientales. Ameghiniana 4: 435-443.

Wood W. y D. Johnson

1978 A survey of disturbance processes in archaeological site formation. Archaeology Method and Theory (ed. por Shiffer M.), pp 315-381. Academic Press, New York.

Zohar I, Dayan T, Galili, E y E, Spanier

2001 Fish processing During de early Holocene: a taphonomic case of study from Costal Israel. Journal of Archaeological Since 28: 1041-1053. 